Meta

Journal des traducteurs

Translators' Journal

\title{
Tournoi pour l'accommodement des dictionnaires de collocations
}

\section{Jean-Luc Descamps}

Volume 39, numéro 4, décembre 1994

Hommage à Bernard Quemada : termes et textes

URI : https://id.erudit.org/iderudit/002545ar

DOI : https://doi.org/10.7202/002545ar

Aller au sommaire du numéro

Éditeur(s)

Les Presses de l'Université de Montréal

ISSN

0026-0452 (imprimé)

1492-1421 (numérique)

Découvrir la revue

Citer cet article

Descamps, J.-L. (1994). Tournoi pour l'accommodement des dictionnaires de collocations. Meta, 39(4), 561-575. https://doi.org/10.7202/002545ar
Résumé de l'article

Cet article montre comment se présentent les collocations du mot joie dans le Trésor de la langue française (vol. X, 1983), le Langenscheidts

Kontextwôrterbuch franzôsisch-deutsch (1989), le Dictionnaire de collocations français-chinois (sous presse) et le Dictionnaire explicatif et combinatoire (vol. 1,1984). Un total de 318 collocations ont été dénombrées sous les articles JOIE de ces quatre répertoires. On en donne la liste alphabétique. Comment l'organiser sémantiquement pour en offrir un accès facile dans un dictionnaire de collocations idéal ? Un défi est lancé aux lecteurs de Meta . [Le concours sera clos le 30 novembre 1995.] 


\title{
TOURNOI POUR L'ACCOMMODEMENT DES DICTIONNAIRES DE COLLOCATIONS
}

JEAN-LUC DESCAMPS

CREDIF, ENS de Fontenay-Saint-Cloud, Saint-Cloud, France

\begin{abstract}
Résumé
Cet article montre comment se présentent les collocations du mot joie dans le Trésor de la langue française (vol. X, 1983), le Langenscheidts Kontextwörterbuch französisch-deutsch (1989), le Dictionnaire de collocations français-chinois (sous presse) et le Dictionnaire explicatif et combinatoire (vol. 1, 1984). Un total de 318 collocations ont été dénombrées sous les articles JOIE de ces quatre répertoires. On en donne la liste alphabétique. Comment l'organiser sémantiquement pour en offrir un accès facile dans un dictionnaire de collocations idéal ? Un défi est lancé aux lecteurs de Meta. [Le concours sera clos le 30 novembre 1995.]
\end{abstract}

\begin{abstract}
This article shows how the collocations of the word joie are presented in the following dictionaries: Trésor de la langue française (vol. X, 1983), Langenscheidts Kontextwörterbuch französisch-deutsch (1989), Dictionnaire de collocations français-chinois (forthcoming) and Dictionnaire explicatif et combinatoire (vol. 1, 1984). A total of 318 collocations are given in these four dictionaries under the headword joie, listed here. The question treated here is this: How can this list be organized semantically so as to give the easiest access to an ideal contextual dictionary? This is the challenge put out to the readers of Meta. Entries to be in by 30 th November 1995.
\end{abstract}

JOIE : la mère des jeux et des ris; on ne doit pas parler de ses filles.

(G. Flaubert, Dictionnaire des idées reçues)

Crainte de déchoir? Manque d'imagination? Les revues savantes n'ont point coutume d'héberger quelque concours que ce soit à l'intention de leurs lecteurs. Voici bientôt quinze ans le directeur des Cahiers de lexicologie en avait pourtant pris le risque en ouvrant sa revue à une sorte de tournoi lexicographique ${ }^{1}$. Dans le présent numéro d'hommage à Bernard Quemada, la revue Meta nous autorise à faire écho à cette fédératrice innovation, et même nous soutient en offrant aux futurs lauréats du tournoi quelques prix promotionnels : qu'elle en soit ici vivement remerciée. Mais de quoi s'agit-il aujourd'hui?

\section{LA NOTION DE COLLOCATION}

Le dictionnaire de collocations a désormais ses lettres de noblesse, puisqu'un article entier de l'Encyclopédie internationale de lexicographie - I (1989)2 lui est dédié, sous la plume de F.-J. Hausmann 3 . À propos de la notion de collocation, adoptons d'emblée pour simplifier les choses - les principes dégagés par l'auteur, soit,'à peu près dans ses termes, les suivants :

[1] La collocation se distingue de la combinaison libre (the book is useful, das Buch ist nützlich, le livre est utile) par la combinabilité restreinte (ou affinité) des mots combinés (feuilleter un livre vs acheter un livre.) Elle se distingue des locutions (idioms, 
Redewendungen, par ex. monter un bateau à qn/jdn. durch den Kakao dringen / to pull sb's legs) par son non-figement et par sa transparence.

[2] Dans la collocation le statut des deux partenaires combinés n'est pas égal. L'un des partenaires est autonome au plan sémantique : on l'appellera base de la collocation; 1'autre ajoute une caractérisation qui ne modifie pas l'identité sémantique du caractérisé : on l'appellera collocatif. On compte en français six structures de collocation, dûment orientées. Exemples - types (base en italique):

- Nom + Adjectif : célibataire endurci

- Nom + Verbe : la colère s'apaise

- Verbe + Adv.: il pleut à verse

- Adv + Adj.: grièvement blessé

- Verbe + Nom : retirer de l'argent

- Nom + prép. + Nom: une bouffée de colère

[3] Parce que les collocatifs doivent être définis, les dicos généraux mentionnent les collocations beaucoup plus aux articles-collocatifs (ex. endurci) qu'aux articles-bases (ex. célibataire). Comme la collocation est avant tout un problème de production de textes (et non de compréhension), le dictionnaire de collocations se doit de mentionner les collocations aux articles-bases.

[4] Sources: faire appel à un corpus (nécessairement immense) tout en s'appuyant sur la compétence des locuteurs est, de fait, impratique. On a donc intérêt à recueillir dans les répertoires existants les collocations déjà recensées soit sous leurs mots-bases soit sous leurs collocatifs.

[5] Les collocatifs du mot-base ont besoin d'être ordonnés et groupés de façon systématique, et le système de critères sémantiques/syntaxiques adapté doit, dans chaque article, être explicité. L'article peut par exemple être balisé à l'aide d'archilexèmes relevés par la typographie ${ }^{4}$ :

Doute: [...] FAIRE NAITTRE : inspirer, EXPRIMER : émettre, formuler; FAIRE DISPARAÎTRE: lever, écarter, éclaircir, dissiper, balayer

[6] Le marquage diasystématique des collocations est indispensable [entendre par là: niveaux de langue, domaines spécialisés, valeurs diachroniques, valeurs évaluatives, etc.].

[7] La distinction entre collocations et combinaisons libres est beaucoup plus incertaine que celle entre collocations et locutions. Aussi faut-il se résigner, en tenant compte de quelques grandes langues de comparaison, à être pragmatique et à n'être pas exhaustif aux confins des combinaisons libres.

Ces principes étant rappelés, et avant d'exposer la matière et l'objectif du tournoi, examinons en bref un échantillon de quatre dictionnaires récents, chacun typique à sa manière, pour leur traitement des collocations.

\section{QUATRE DICTIONNAIRES SUR LE GRIL}

À titre d'exemple, concentrons-nous sur un seul mot, en l'occurrence, le mot joie, et ouvrons à l'article JOIE un grand dictionnaire général, le Trésor de la langue française $(T L F)^{5}$, et trois dictionnaires spécialisés, dont deux bilingues et un monolingue, resp.: le Kontextwörterbuch Französisch - Deutsch $(K F D)^{6}$, le Dictionnaire des collocations français-chinois $(D C F C)^{7}$, et le Dictionnaire explicatif et combinatoire du français contemporain $(D E C)^{8}$. Combien y peut-on compter de collocations de JOIE $(\$ 2.1$.), et comment y sont-elles présentées $(\S 2.2$.$) ?$

\subsection{Tableaux quantitatifs}

A) Tableau général [318 collocations]:

a) 208 colloc. propres à 1 dico 81 colloc. propres à 2 dicos 26 colloc. propres à 3 dicos 3 colloc. communes à 4 dicos
B) $K F D: 42$ (dont 7 propres)

$T L F: 107$ (dont 52 propres)

$D C F C: 140$ (dont 62 propres)

$D E C: 171$ (dont 87 propres) 
B) Tableaux par structures:

a) 189 collocations avec verbes (ex : crier de joie /la joie l'inonde) :

a) 125 colloc. propres à 1 dico 52 colloc. propres à 2 dicos 11 colloc. propres à 3 dicos 1 colloc. communes à 4 dicos

$\beta) K F D: 18$ (dont 3 propres) $T L F: 50$ (dont 22 propres) $D C F C: 88$ (dont 45 propres) $D E C: 110$ (dont 55 propres)

b) 112 collocations avec adjectifs (ex. joie débordante /fausse joie) :

a) 70 colloc. propres à 1 dico 25 colloc. propres à 2 dicos 15 colloc. propres à 3 dicos 2 colloc. communes à 4 dicos
B) $K F D: 24$ (dont 4 propres) $T L F: 46$ (dont 23 propres) $D C F C: 52$ (dont 17 propres) $D E C: 51$ (dont 26 propres)

c) 17 collocations avec $\mathrm{N}+$ prép + joie (ex : sentiment de joie) :

$T L F: 11$ (dont 4 partagées avec le $D E C$ )

$D E C: 10$ (dont 4 partagées avec le $T L F$ )

[NB : aucune dans $K F D$ et dans $D C F C$ ].

\subsection{Présentation des collocations}

A) Dans le Trésor de la langue française (TLF):

À tout seigneur tout honneur: commençons par ce grand édifice, élevé sous la direction de Paul Imbs, puis de Bernard Quemada, et dont le dernier volume se publie cette année. Dans ce répertoire général, le seul de notre échantillon, nous avons compté 107 collocations différentes. Entendons ici: sous l'article JOIE, car maintes autres s'y ajouteraient si nous chalutions les articles possiblement collocatifs (ex. briller de joie sous BRILLER, cacher sa joie sous CACHER, etc.), et la masse des exemples disséminés dans tout le $T L F$... Quoi qu'il en soit, de ces 107 collocations de l'article JOIE, 26 figurent groupées sous une rubrique SYNTAGME réservée à cet effet (en queue de rubrique Av. squelette de l'article (fig.1), ci-après). Les 81 autres, la majorité donc, sont réparties au fil des rubriques et sous-rubriques: soit par petites escouades ou singulets non référencés; soit isolément dans des exemples référencés, où nous sommes allés les repêcher à nos risques et périls (NB : les «Normes» du TLF en effet imposaient censément aux rédacteurs de ne pas répéter dans les exemples référencés les collocations déjà mentionnées sans référence). Certes, nous avons eu quelques hésitations lors de ce repêchage. Elles sont cependant de peu de poids aujourd 'hui. C'est qu'en effet notre tournoi, nous y reviendrons, va porter sur un problème limité : non pas sur celui du départage entre collocations et combinaisons libres (ou entre collocations et locutions rigides); mais sur celui - une liste de collocations étant admise en tant que telles sous tel article de tel dictionnaire spécifique - de leur commodité d'accès sémantique pour un rédacteur et, singulièrement, pour un traducteur.

De ce point de vue nous ne saurions sans injustice juger du $T L F$ - ne serait-ce même qu'à l'article JOIE - puisque ce répertoire n'est pas à proprement parler un dictionnaire spécifique «de collocations». Il faut noter certes l'agrément de la rubrique SYNTAGME, et le naturel avec lequel s'offrent en lecture continue les collocations au fil de l'article. Mais on peut estimer qu'un rédacteur moyen éprouverait sans doute quelque gêne à se piloter vivement vers la rubrique la plus apte à lui fournir les collocations répondant à ses besoins. Pour nous aviser, au reste, de la difficulté, il n'est que de nous essayer au mouvement inverse que voici : replacer dans les diverses rubriques de l'article du TLF ci-après réduit à ses définitions (fig.1) - les collocations qui y étaient hébergées (celles marquées d'un T dans la liste des données, v. infra, § 3.): cet exercice a tergo nous vaudrait assurément quelques tracas. S'en étonner cependant messierait: le $T L F$, répétons-le, est 
un répertoire n'appartenant pas à la catégorie des dictionnaires de collocations - celle qui va désormais retenir notre attention.

Fig. 1:

Squelette de l'article JOIE du $T L F$

A. - Émotion vive, agréable, limitée dans le temps; sentiment de plénitude qui affecte l'être entier au moment où ses aspirations, ses trésors ou ses rêves viennent à être satisfaits d'une manière effective ou imaginaire.

1. [le sentiment est d'ordre affectif, moral, intellectuel ou psychique]. Contentement, profonde satisfaction [exemples].

- Locutions [exemples].

- En particulier

- [La joie est vécue dans une relation avec une autre pers.] [exemples].

- [La joie est vécue dans une relation avec plus. pers., un groupe] [exemples].

- [Avec une valeur négative; le sentiment de joie est altéré par les mauvais sentiments (envie, haine, malveillance,...)] [exemples].

2. [Le sentiment de joie affecte l'humeur]. Entrain, gaieté. [exemples].

- Locutions [exemples].

3. [La joie est considérée dans son origine]

a) [La cause de la joie est dans le corps, les sens]. Plaisir.

- [sensualité] [ex.] • locution [ex.]

- [sexualité] [ex.] • locution [ex.]

b) [La cause de la joie a sa source dans la nature, dans la vision de la beauté] (ex.)

c) [La cause de la joie réside dans la réalisation d'un projet, d'un désir, d'un rêve] (ex.).

d) En partic. Joie de vivre. Sentiment de bien-être général, de bonheur complet qui vient du simple fait d'exister [ex.].

SYNTAGMES (26).

B. - [Cet état de sensibilité considéré dans ses expressions les plus spectaculaires et le plus souvent collectives]. Manifestation dé gaieté, de liesse, d'allégresse publique [ex.]

- Locutions [ex.].

C. - [Le sentiment de joie au-dessus de tout, préférable à tout]. Bien suprême [ex].

- Locutions [ex.].

- [Mystique religieuse] [ex.].

- Par métonymie :

- [En parlant d'une personne qui est la source de joie pour qqn; personne qui incarne cette joie [ex.].

- [Plus rare. En parlant d'un animal ou d'une chose qui est source de joie pour qqn [ex.].

B) Dans le $K F D$, dictionnaire de collocations français-allemand :

Publié en 1989, ce répertoire utilitaire de 320 pages, à l'usage des Allemands, affiche quelque 20000 collocations réparties sous 3500 vocables substantifs environ. Chaque article présente successivement: d'abord la base et sa traduction en allemand; puis, les collocations à collocatif adjectif, également traduites; enfin, les collocations à collocatif verbe, traduites et de surcroît illustrées pour la plupart d'un exemple en français. Nous n'avons observé ni collocations de Type $N+$ prep, ni a fortiori de type $V+a d v$ ou $a d v$. adj. Sous un article donné - et ce en chacun des deux ensembles adjectivaux puis verbaux - les collocations se suivent dans l'ordre alphabétique des collocatifs; ceux-ci figurent parfois en paires ou triolets lorsque les collocations où ils entrent sont estimées synonymes (ex. joie cachée, secrète; ou bien: sauter, bondir, danser de joie). Dans les cas saillants, les singularités syntaxiques (ex.: "jour .... couler des jours" (+ adj.)) ou même diasysté- 
matiques (ex : «colle (Schülersprache) :... poser une colle...» [NB. Schülersprache $=$ argot scolaire, trad. J.L.D.]) sont signalées. Quant à l'cil du consultant, il est guidé à merveille par une typographie bien contrastée. Exemple: photo de l'article JOIE du KFD ci-dessous (fig. 2).

Fig. 2 :

Article JOIE du $K F D$

jole $f$ Frende

antidpte Vorfreude / - bruyante selrmische Freude I d debordante, folle uberschäumende Freude; Freudentaumel T cachte, secrite heimliche Preude / - dellirante, effrtnée wahnismige, unbändige Freude; Freudenrausch I - Etudlte geküntelke, geheuchelte Freude / excessive maplose Freude I - exuberante überscheoengliche Freude / fausse - verfrithte Freude / Immense ; intense sehr große, riesige Freude / indtcente schamlose Freude / - indicible, ineffable unaussprechliche, unsagbare Freude / - insolente wrverschämte

Freude I - mallgme; mauvaise Schadenfreude; hämische Freude / muette stille, innere Freude / profonde innige Freude / satanique teuffisches Vergnigen / - sereine ungetribte Freude

combler, remplir qn de $\sim j-n$ mit Freude erfüllen: Certe nouvelle amblé de $\sim$ la paurre condamnece. / donner de la $\sim$ Freude bereiten, machen: Voure livre m'a donne de la $\sim$ / empoisonner, troubler la $\sim$ de qn $j$-s Freude trüben: Un soupcon sans fondement empoi-

\begin{abstract}
sonne $30 \sim$ depuis un certain ternps. / Éprouver, ressendir de la $\sim$ Freude empfinden: En lisent votre lettre, $j$ 'ai éprouvé une très grande - / trve en vergnügt, ausgelassen sein: Après un bon apéritif, tout le monde était en $\sim 1$ faire la w tare la seule $\sim$ de qn $j-s$ ganze Freude sein: Son fils faiseit sa seule ... / se faire une $\sim$ de faire qe sich ein Vergniugen daraus machen, etwo zu tun: Elle se fait une $\sim$ d'alles travaillex une annece aux Etats-Unis. / mettre qn $\mathrm{cn} \sim j$ jn erheicerm, fröhlich stimmen: Pierre Leclancher est très vite parvenu à mettre son auditoire en $\sim$ / partager la de an $j$-s Freude teilen: Nous partageons votre $\sim$. / rayonner de - (vor Freude) strahlen: Son visage rayonnait de - / sauter, bondir, danser de $\sim$ Freudenspringe machen: Les enfants ont sauté de en entendant que leur père avait acheté un chalet. / être transporté de vor Freude ganz weg sein: Lorsqu'il a appris la nouvelle, il a èté transporté de .
\end{abstract}

Au total, cet ouvrage modeste et efficace présente des qualités appropriées à la clientèle visée : celle de rédacteurs et traducteurs de niveau convenable, ou s'exerçant à y atteindre. On peut néanmoins avancer deux remarques. La première, au reste sans rapport avec notre tournoi, est que l'ouvrage aurait pu utilement faire précéder les exemples en français de leur traduction en allemand: l'apprenant autonome et de tempérament volontariste aurait pu ainsi commodément s'en servir comme d'un manuel d'exercice de thème (hinübersetzen); mais, nous en convenons, cela eût enchéri l'ouvrage... La seconde remarque en revanche touche l'objet de notre concours, à savoir, nous l'avons dit, la commodité d'accès vers les collocations idoines au besoin du rédacteur à l'instant $t$. L'ordre alphabétique sur lequel se fonde le $K F D$ convient, certes, mais seulement dans la mesure où l'œil n'a pas à parcourir une liste trop longue. C'est le cas de JOIE dans le $K F D$. Mais cette liste aurait pu être beaucoup plus étoffée, puisque nous avons compté, sommant les quatre dictionnaires de l'échantillon, plus de 300 collocations différentes. Avec un tel nombre, un classement réglé par le sens n'eût-il pas été plus efficace ?

C) Dans le $D C F C$, dictionnaire de collocations français-chinois :

Il s'agit, comme le $K F D$, d'un répertoire utilitaire, cette fois à l'usage du rédacteur chinois. De rédaction achevée et voué à prochaine publication en Chine, il affiche sensiblement moins de bases que son homologue allemand ( 2450 , contre 3500 ), mais, soulignons-le, beaucoup plus de collocations ( 54600 , contre 20000 dans le $K F D$ ). Les articles, en majorité à base nominale, présentent en succession: d'abord la base et sa traduction; puis les collocations à collocatif verbal, traduites en chinois (mais, à la différence du $K F D$, non illustrées d'exemples en français); enfin celles à collocatif adjectival, elles aussi 
traduites. La rubrique ÉPITH qui regroupe ces dernières - en général dans l'ordre alphabétique - affiche aussi, quoiqu'irrégulièrement, des structures de type : $N+$ prép $+*$ (ex. «haine [...] ferments, sources, levains de *), ou de type: * + prép $+N$ (ex. «accident [...] * de la route, * tragique, * du travail). Outre les articles à base nominale figurent aussi un nombre non négligeable d'articles à base verbe, affichant et traduisant les collocations de type $V+a d v$. Ex «accepter.: [trad]. * de grand cour, avec plaisir: [trad.]; * avec empressement [trad.]; * en toute confiance, (1. p.) *les yeux fermés [trad.]; * à contre-cceur [trad.]. Enfin, maintes particularités syntaxiques sont indiquées (ex. «aide [...] : accorder une ${ }^{*}+$ adj. à qn [...]»; ex. joie [...]: qc manifeste, montre, exprime la ${ }^{*}$ de qn [...]). Quant aux phénomènes diasystématiques ou aux traits d'intensité (au moyen de flèches $\uparrow$ ou $\downarrow$ ), s'ils faisaient partie du programme lexicographique de l'ouvrage, ils n'ont en fait été notés qu'occasionnellement, à en juger d'après le manuscrit entre nos mains. Pour ce qui est des traductions, elles ont forme de collocations, de combinaisons libres ou d'interprétations selon le cas, comme celles du KFD.

Hormis les différences de nomenclatures et d'effectifs, deux traits distinguent surtout, à nos yeux, le $D C F C$ de son cousin... germain. Le premier est à l'avantage du $K F D$, seul des deux répertoires à illustrer ses collocations verbales d'exemples phrastiques, certes non référencés, mais fort naturellement tournés. Le second trait, quant à lui, fait honneur à la hardiesse du $D C F C$ : il touche l'ordre de succession des collocations verbales au sein de chaque article, autant dire l'enjeu même de notre tournoi. En effet, nul ordre alphabétique n'ordonne la file de ces collocations mais un ordre - autant que faire se peut - sémantique. Principale hypothèse de travail des auteurs: sous le gros des bases de la nomenclature - c'est-à-dire les bases désignant des états, des événements ou des actions — il serait d'ordinaire possible de disposer les collocations verbales en cycle, de sorte qu'apparaisse l'évolution logique de la notion désignée par la base, «avec sa naissance, son développement, son apogée, son déclin, sa disparition et enfin sa renaissance éventuelle» (Liang 1991) ${ }^{9}$; au besoin, on regrouperait localement les collocations quasi synonymes. L'hypothèse a effectivement été éprouvée sur l'ensemble de la nomenclature - non sans d'indiscutables résultats, certes, mais non sans à peu près, et non sans laisser finalement au lecteur le sentiment que de nombreux problèmes sont restés en suspens. Dans l'urgence de leur tâche, les auteurs n'ont d'ailleurs à aucun moment cherché à se rapprocher de la maxime idéale proposée dans l'Encyclop. Internat. de lexicogr. (op. cit.), à savoir celle d'un balisage explicite qui eût justifié l'emplacement occupé par telle et telle collocation en chaque article et qui eût étiqueté leur valeur sémantique. On reproduit ci-contre (fig. 3), à titre d'exemple, l'article JOIE du DCFC [v. aussi l'article HAINE in Liang, op. cit.).

Fig. 3:

Article JOIE du $D C F C$

joie

1. 欢乐, 高兴, 喜悦.

inspirer. causer. apporter. procurer. donner de la -- à qn: provoquer la --- de/chez qn; emplir, remplir qn de --- : mettre qn en --.- 令人高兴 la --- s'empare de. envahit, pénètre, saisit qn某人一阵欣喜être en --: être saisi de -.- 心中高兴 éprourer, (litt.) concevoir. sentir, ressentir, aroir de la --. 感到高兴manifester. montrer, té moigner, exprimer, exté rioriser, é pancher. exhaler sa --- 表示高兴, 流露出喜悦心情 (qch) manifeste. montre. exprime la $\cdots$ de qn (某事) 表现出某人的喜悦 la --- se manifeste. parait. se peint. se lit .éclate $\uparrow$. explose le visage de qn 喜形于色 la -.- illumine, fait rayonner le visage de qn 某人满面春风 (qn/qch) répandre la --- 散布欢乐情绪 être gonflé. comblé. rempli, pé né tré de --- 内心充满喜悦 la --- inonde le coeur de qn 满心欢喜 (qn) baigne. nage dans la --- 沉浸于欢乐之中 déborder. être inondé, rayonnant de -.- 
洋溢着欢乐 délirer $\uparrow$, exulter $\uparrow$, éperdu. transporté. (fam.)fou, ivre $\uparrow$ de --. 欢喜若狂 palpiter de -.- 高兴得心直跳 s'abandonner. se livrer. se laisser aller, laisser libre cours à la --. 尽情欢乐 défaillir, ne plus sentir. ne plus se possé der de - 喜不自禁, 乐不可支 la -- pétille, brille dans ses yeux: (veux. regard) briller. pétiller. étinceler de -.. 眼睛闪炼着喜悦的光芒 pleurer de -.. 高兴得哭了 être ému de -.. 高兴得激动不已 la -.. é gare qn 高兴得忘乎所以 frétiller de -... 高兴得坐不住了 bondir. sauter de ... 高兴得跳起来 gambader de ... 高兴得直蹦 (指小孩) se pâmer de ... 欢喜欲绝 frémir. tressaillir de ... 高兴得罟抖不已 tré pigner de -.. 高兴得手舞足蹈 ètre au comble de la -... 极端喜悦 redoubler la -... de qn 使某人倍增喜悦 confier à qn sa -.. 向某人吐露心中的喜悦 communiquer sa -... à qn 把高兴的心情感染别人 ć prourcr une --- mê lé e de tristesse; ê tre partagé entre la --- et la peine: la --- est mê lé e de tristesse 悲喜交加 la -- se change en tristesse 乐极生悲 entrer dans. prendre part, participer à la -.- de qn; partager la (les) --- de qn 分章某人的欢乐 partager les ---s et les peines de qn 与某人同甘共苦 affecter. feindre la ... 假装高兴 cacher. (dissimuler) sa -.- 掩盖, (掩饰)高兴的心情 contenir sa --. 抑制住内心的喜悦 gâcher. ternir la -.. de qn 破坏某人的欢乐ôter la ... a qn 使某人失去欢乐 accepter qch. avec 高兴地接受某物

Epith. --- ardente 热烈的高兴心情 -.. austè re 严肃的欢乐 -.. bruvante. dé lirante. extatique. folle. turbulente 狂欢:狂喜 ... calme. sereine 恬静的喜悦心情 ... communicative 感染人的欢乐心情 -..- complè te. sans mélange, pure, parfaite 没有一丝阴影的欢乐情绪 -... e phémè re. courte. passagè re短暂的欢乐 -... étudić e. fcinte 做作的喜悦 -.. excessive. immodé ré e 过分高兴 ... extraordinaire 异常高兴 --. extrê me, féroce. insigne. intensc. infinic. profonde. grande. vire 极端高兴 -.- exubérante 溢于言表的喜悦 faussc -... 空欢喜 -... gé né rale 普遍的高兴 ... incomparable 无比高兴 ... indescriptible. 敒exprimable. ineffable. indiciblc 说不出的高兴:无以名状的喜悦 -.. incroyble 难以想象的高兴 -.. insolcnte 过分外露的高兴 … intime inte rieure 内心的喜悦 … maligne. satanique 狞喜 -.. muette 无声的喜悦暗喜-.. puérile 象小孩似的高兴 -.. rayonnante. radieuse 喜气洋洋 -.- tendre 淡淡的高兴心情

2. 乐趣,乐事令人高兴的事

être, faire la -.- de qn 是某人的快乐, 是某人的慰籍 se faire une --. aroir la --. de f. qch. 乐于干某事 (qch.) renferme des --.s (某事)具有乐趣

Epith. memtes. petites ---s 小乐趣 ---s intarissables, inepuisables无穷的乐趣

D) Dans le $D E C$, dictionnaire explicatif et combinatoire du français contemporain :

Il s'agit ici, soulignons-le d'emblée, d'un répertoire dont la visée, l'assiette et la teneur diffèrent radicalement de celles des deux «dictionnaires de collocations» ci-avant examinés.

La visée? Le $D E C$ n'est pas un dictionnaire utilitaire, prévu pour une clientèle d'apprenants, de rédacteurs ou de traducteurs; c'est un répertoire à vocation scientifique, ce que manifeste en clair le sous-titre de l'ouvrage: «Recherches lexico-sémantiques».

L'assiette? Le $D E C$ est conçu selon les auteurs comme «partie intégrante d'une description théorique d'une langue naturelle». Il s'agit en l'occurrence du modèle linguistique dit «sens-texte», selon lequel une langue donnée est un système de règles assurant correspondance entre l'ensemble infini des sens et l'ensemble infini des textes de cette langue: «le dictionnaire de cette langue doit donc fournir au système, sur les mots individuels, toutes les données indispensables à son fonctionnement» (DEC 1, op. cit. p. 3). 
La teneur? Ce qui vient d'être dit laisse présager que le $D E C$ est beaucoup plus qu'un dictionnaire de collocations stricto sensu et, de fait, il met en œuvre un programme lexicographique d'une tout autre ampleur. Pour chaque sens (ou lexème) distingué sous chaque mot (ou vocable) de la nomenclature, ce programme aligne en effet régulièrement quatre parties: définition, schémas de régime, fonctions lexicales, et exemples phrastiques. C'est d'ailleurs, nous y reviendrons, sous la partie vouée aux fonctions lexicales que se trouvent dûment distinguées et analysées - outre les synonymes, antonymes, dérivés, génériques, conversifs et autres - les collocations, au sens hausmannien ici adopté. Touchant la teneur, concluons cependant par quelques chiffres. La nomenclature des trois volumes à ce jour publiés compte quelque 270 vocables (resp. vol. 1 [1984]: 50; vol. 2 [1988] : 107; vol. 3 [1992]: 110). C'est peu auprès de celle du KFD (3500 vocables) ou même du $D C F C$ (2450), ce d'autant qu'il s'agit d'une nomenclature ouverte: noms, certes, mais aussi verbes, adverbes, adjectifs et locutions diverses. Néanmoins, explorant comme elle le fait une diversité choisie de champs sémantiques (parties du corps, météo, éducation, mots locutoires, sentiments, modalité déontique, commerce, ...), c'est une nomenclature d'ampleur suffisante pour apprécier le fort et le faible du projet. Enfin, pour ce qui est des collocations, leur nombre commande le respect et l'emporte constamment - souvent de beaucoup - sur le nombre qu'affichent sous un même vocable ses compétiteurs.

Qu'en est-il cependant, car c'est le point de mire du tournoi, de l'ordonnancement et de la présentation qualitative des collocations dans le $D E C$ ?

L'ouvrage les décrit, signalions-nous supra, au chapitre et dans le cadre des fonctions lexicales. Qu'est-ce à dire en l'espèce ? À propos de fonction, nous avons tous en tête la formule classique: $\mathbf{f}(x)=y$, laquelle pointe sur une certaine valeur $y$, obtenue, elle, au terme d'une opération $\mathbf{f}$ exécutée sur une certaine valeur $x$. Appliquons à cette formule la terminologie hausmannienne: on a alors: $\mathbf{f}($ base $)=$ collocatif. Plus précisément: fixons $\mathbf{x}$, en lui imposant la valeur lexicale : joie. On a: $\mathbf{f}($ joie $)=$ collocatif. Explorons à présent quelques opérations $\mathbf{f}$ applicables à joie:

exemple 1:: supposons que $\mathbf{f}=$ porté à un degré élevé : quelle peut bien être alors la valeur de $y$ ? En d'autres termes, comment la langue accoutume-t-elle de dire: «une joie portée à un degré élevé»? Une poignée de qualifiants nous vient spontanément à l'esprit, postables avant ou après joie selon le cas: grande, immense, extrême, indescriptible, vive, profonde, sans bornes... En remplaçant porté à un degré élevé, trop long, par l'abréviation symbolique Magn, notre formule devient: Magn (joie) = grande, immense, extrême, vive [etc.].

- exemple 2 : supposons que $\mathbf{f}=$ verbe qui, sans nuance particulière, admet comme complément le mot joie et comme sujet la personne joyeuse : quelle peut bien être alors la valeur de $y$ ? Là encore, quelques verbes typiques nous viennent à la bouche (éprouver, ressentir, avoir...). En remplaçant verbe qui [etc] par l'abréviation symbolique Oper 1 , notre formule s'écrit: Oper 1 (joie) = éprouver, ressentir, avoir. [NB : L'indice bas 1 affecté à Oper permet de signaler que le verbe $x$ a pour sujet grammatical le premier actant sémantique de la situation exprimée par le mot joie, $\mathrm{c}$.-à-d. ici la personne qui est siège de l'émotion].

- exemple 3: supposons que $\mathbf{f}=$ verbe qui exprime le rapport précédent (à savoir Oper $_{1}$ ) associé à l'idée qu'il s'agit d'une joie portée à un degré élevé. Par association de symboles, on peut alors livrer les valeurs banales qui viennent à la plume ou au gosier: Magn + Oper 1 (joie) $=$ nager, baigner [dans la $*]$, exulter $[d e *]$. Et on peut même y adjoindre telles expressions figées exprimant synthétiquement la même idée (sans même qu'y apparaisse le mot joie) et les munir au besoin de traits diasystématiques, ce qui donne, signalé dans le $D E C$ par double barre oblique : // fam. être aux anges, fam. être au septième ciel.

- exemple 4: supposons maintenant que $\mathbf{f}=$ verbe qui admet joie comme sujet [ce que rendra le symbole Func], la personne joyeuse comme complément [ce que rendra l'indice bas 1], associé à l'idée inchoative [ce qui rendra le préfixe symbolique Incep]. Le $D E C$ rendra alors compte des collocations possibles sous la forme: Incep Func 1 (joie) $=s^{\prime}$ emparer [de $N]$; saisir, gagner [N] ; naitre [en son cour, âme]. 
Nous venons d'expliciter quatre rubriquettes du $D E C$ livrant, au chapitre des fonctions lexicales et sous le lexème JOIE 1, une vingtaine de collocations. Nous avons tenté ainsi de fournir un aperçu des principes d'écriture des collocations dans l'ouvrage. Donnons encore quelques précisions, quantitatives notamment. L'ensemble des fonctions lexicales (synonymies, antonymies, dérivés [etc.] et collocations) s'exprime dans le $D E C$ à l'aide d'une cinquantaine de symboles simples (ex. Magn, Oper, Func,...). D'aucuns peuvent se trouver munis d'indices (ex. Oper ${ }_{1}$, Func $_{0}$, Magn temp,,.. ), voire soudés (ex. IncepFunc $_{1}$, CausPredMinus,...) ou additionnés (ex. Magn + Oper 1 ). En outre, il arrive que, faute de symboles standard adéquats, certaines collocations soient signalées par des formules non standard (ex. tel que y est un mal pour qn (joie) = méchante, mauvaise $<$ cruelle sadique). On compte, sous l'article JOIE (c.-à-d. sous les trois lexèmes JOIE 1 , JOIE 2 , JOIE $_{3}{ }^{10}$ ), un total d'environ 171 collocations hausmanniennes, distribuées sous quelque 70 rubriquettes du genre de celles que nous avons glosées plus haut. Quelque vingt-sept symboles sont itérativement utilisés, et une douzaine de formules non standard.

On a reproduit en note 13 (v. infra) un fragment continu et significatif de la section des fonctions lexicales listées sous le lexème JOIE $_{1}$. On y compte 25 rubriquettes livrant en dominante des collocations à collocatifs verbaux (ex : éprouver, perdre, etc.) et adjectivaux (ex. sereine, sans mélange, etc.), mais aussi des syntagmes ne répondant pas à la définition hausmannienne des collocations (ex. à la joie de, de joie,...). Ce fragment permet incidemment de relever une défaillance régulière du $D E C$ touchant les collocations avec verbes: leurs transformations nominales acceptables ou inacceptables ne sont point indiquées [ex : disparaittre [disparition], s'évanouir [*évanouissement], s'éteindre [*extinction], cesser [*cessation]. Mais cette carence relative importe peu ici, le fragment reproduit devant surtout nous permettre de cerner un problème bientôt crucial pour notre tournoi : celui de l'ordre de présentation des collocations. Les auteurs énoncent à ce propos un principe important: en tête de liste figurent les synonymes, conversifs, antonymes, génériques, et dérivés syntaxiques; viennent ensuite le reste des fonctions lexicales. La plupart des collocations hausmanniennes se trouvent donc parmi ce «reste», d'ailleurs considérable. Les auteurs précisent encore : d'une part, que, d'après leur partie du discours, les fonctions lexicales se suivent selon l'ordre noms, adjectifs, adverbes, verbes; d'autre part, qu'une collocation plus abstraite (ex. Oper) doit censément précéder une fonction lexicale plus concrète (ex Fact, Real) $[D E C$ 1:7]; mais ces deux principes hiérarchiques ne semblent régir que des ordres locaux.

C'est, au total, cornucopie de collocations qu'expose le DEC à son étal - si court soit-il - et l'analyse structurée qui en est faite pique constamment le linguiste et le didacticien ${ }^{11}$. Il reste que la forme de cette analyse est savante; que l'esprit se doit en bonne justice de l'assimiler «à la rigueur», comme eût dit Saniette au grand courroux de Monsieur Verdurin'2, mais que l'œil en vérité ne s'y meut lestement ni de gauche à droite, ni de haut en bas, comme le fait d'ailleurs observer F.-J. Hausmann (op. cit: 1012). D'où l'évaluation, crûment formulée par notre cousin, traducteur de son état: «L'accès aux collocations dans le $D E C$, c'est pas de la tarte !» Et cela nous ramène droit à notre tournoi, dont nous exposerons maintenant la matière et les règles.

\section{MATIÈRE ET RÈGLES DU TOURNOI}

\subsection{La matière du tournoi}

Il va s'agir pour les concurrents de «travailler» la liste suivante, laquelle alphabétise au mot le plus saillant l'ensemble des 318 collocations différentes relevées aux articles JOIE des quatre répertoires de référence. Pour l'aisance du «travail», on a numéroté continûment ces collocations de 1 à 318 , et on a divisé cette liste en trois : A) collocations à 
collocatif verbal ( $\mathrm{N}_{\mathrm{o}} \mathrm{V}$ et $\mathrm{VN}_{1}$, celles de type $\mathrm{N}_{\mathrm{o}} \mathrm{V}$ étant marquées d'un o antéposé); B) collocations à collocatif adjectif; $\mathrm{C}$ ) collocations $\mathrm{N}+$ prép + joie.

La présence de telle collocation dans tel des quatre articles est signalée par un $\mathrm{K}$ pour le $K F D$, un $\mathrm{C}$ pour le $D C F C$, un $\mathrm{T}$ pour le $T L F$, et un $\mathrm{M}$ (pour le $D E C$ publié à Montréal, à la $M A G N$ sueur et mâle myopie d'Igor Mel'čuk).

Abréviations :

\section{LISTE DES COLLOCATIONS DE JOIE}

$\mathrm{K}=$ Langenscheits Kontextwörterbuch Französisch-Deutsch

$\mathrm{C}=$ Dictionnaire français-chinois des collocations du français

$\mathrm{T}=$ Trésor de la langue française

$\mathrm{M}=$ Dictionnaire explicatif et combinatoire du français contemporain

$\mathrm{o}=$ Collocations de type $\mathrm{N}_{\mathrm{o}} \mathrm{V}$

A) Collocations à collocatif verbal:

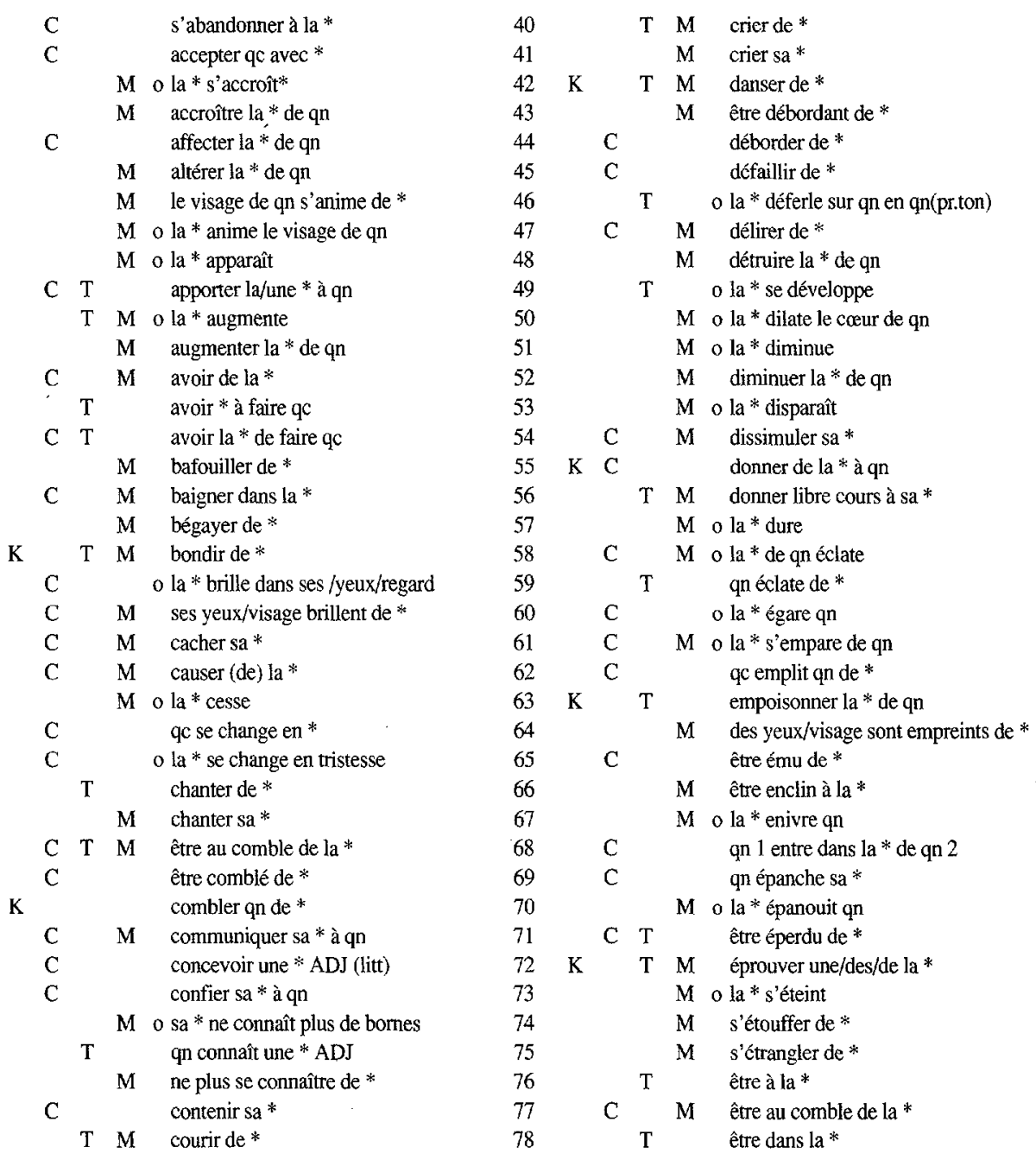




\begin{tabular}{|c|c|c|c|c|}
\hline $\mathbf{K}$ & & $\mathrm{T}$ & $\mathbf{M}$ & être en * \\
\hline & & $\mathbf{T}$ & & être toute la * de qn \\
\hline $\mathbf{K}$ & & & & être la seule ${ }^{*}$ de qn \\
\hline & & & $\mathbf{M}$ & être tout à la * \\
\hline & & & $\mathbf{M}$ & o la * s'évanouit \\
\hline & $\mathrm{C}$ & & & qn exhale sa * \\
\hline & & & M & o la* de qn explose \\
\hline & $\mathrm{C}$ & & $\mathbf{M}$ & $q c$ exprime la * de $q n$ \\
\hline & $\mathrm{C}$ & & $\mathbf{M}$ & qn exprime sa * \\
\hline & $\mathrm{C}$ & & & qn extériorise sa* \\
\hline & $\mathrm{C}$ & $\mathrm{T}$ & $\mathbf{M}$ & exulter de * \\
\hline & & & $\mathbf{M}$ & o la/ma * faiblit \\
\hline K & $\mathrm{C}$ & $\mathrm{T}$ & $\mathbf{M}$ & faire la $a^{*}$ de qn \\
\hline $\mathbf{K}$ & $\mathrm{C}$ & & & se faire une ${ }^{*}$ de $q c$ \\
\hline & & $\mathrm{T}$ & & ça me fait $*$ de faire qc \\
\hline & $\mathrm{C}$ & & & feindre la * \\
\hline & & $\mathrm{T}$ & & fondre de * \\
\hline & C & & $\mathbf{M}$ & être fou de * \\
\hline & $\mathrm{C}$ & $\mathrm{T}$ & M & frémir de * \\
\hline & $\mathrm{C}$ & & & frétiller de* \\
\hline & $\mathrm{C}$ & & $\mathbf{M}$ & gâcher la * de qn \\
\hline & & $\mathbf{M}$ & 0 & la * gagne qn \\
\hline & & $\mathbf{M}$ & 0 & la * grandit \\
\hline & & M & 0 & la * habite qn/qp \\
\hline & $\mathrm{C}$ & & 0 & la $*$ illumine le regard/les yeux de qn \\
\hline & $\mathrm{C}$ & & $\mathbf{M}$ & o la * inonde (le cceur de) qn \\
\hline & & & $\mathbf{M}$ & o la * inonde (le visage de) qn \\
\hline & & & M & qc inonde qn de $*$ \\
\hline & $\mathrm{C}$ & $\mathrm{T}$ & & être inondé de * \\
\hline & $\mathrm{C}$ & & & inspirer de la $*$ à $q n$ \\
\hline & $\mathrm{C}$ & & M & être ivre de * \\
\hline & & & M & o la * de qn jaillit \\
\hline & & & $\mathbf{M}$ & jeter une ombre sur la * de qn \\
\hline & & $\mathrm{T}$ & & être un jour de * \\
\hline & $\mathrm{C}$ & & $\mathbf{M}$ & se laisser aller à la * \\
\hline & $\mathrm{C}$ & & & laisser libre cours à sa * \\
\hline & $\mathrm{C}$ & & & o la * se lit sur le visage dè qn \\
\hline & $\mathrm{C}$ & & & se livrer à la * \\
\hline & $\mathrm{C}$ & & & qc manifeste la * de qn \\
\hline & $\mathrm{C}$ & & $\mathbf{M}$ & qn manifeste sa * \\
\hline & $\mathrm{C}$ & & M & une * mêlée de tristesse/larmes \\
\hline & & & $\mathbf{M}$ & se mettre en * \\
\hline & & & $\mathrm{M}$ & mettre fin à la* de qn \\
\hline $\mathrm{K}$ & & $\mathbf{T}$ & M & mettre qu en * \\
\hline & & & M & qc montre la $*$ de qn \\
\hline & C & & $\mathbf{M}$ & qn montrer sa * \\
\hline & C & & $\mathbf{M}$ & nager dans le * \\
\hline & & & $\mathrm{M}$ & o la * naît dans l'âme/le couur de qn \\
\hline & & & $\mathrm{M}$ & être pétulant de * \\
\hline & $\mathrm{C}$ & & & ôter la *à qu \\
\hline & $\mathrm{C}$ & & & palpiter de * \\
\hline & $\mathrm{C}$ & & $\mathbf{M}$ & se pâmer de * \\
\hline & $\mathrm{C}$ & & & être partagé entre la * et la peine \\
\hline $\mathbf{K}$ & & & $\mathbf{M}$ & partager la * de qn \\
\hline & $\mathrm{C}$ & & & partager les * et les peines de qn \\
\hline & $\mathrm{C}$ & & & participer à la * de qn \\
\hline
\end{tabular}

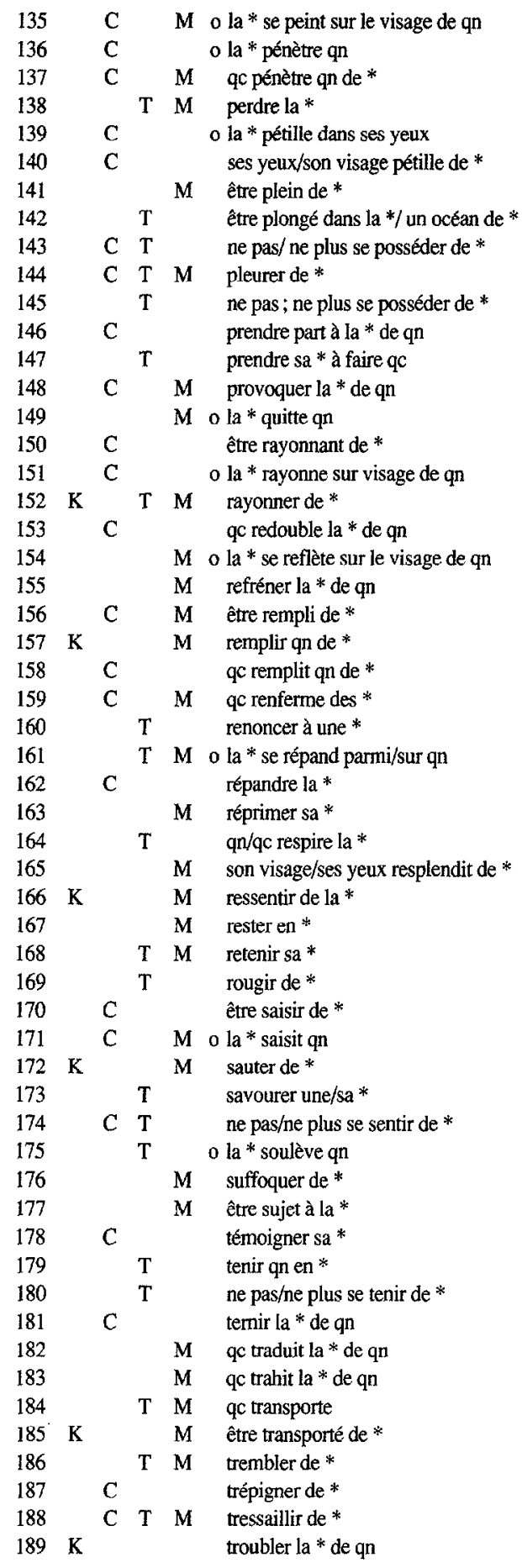


B) Collocations à collocatif adjectif :

\begin{tabular}{|c|c|c|c|c|c|c|c|c|c|c|}
\hline 190 & & $\mathrm{~T}$ & $M$ & * amère & 244 & & $\mathrm{C}$ & & & * incroyable \\
\hline 191 & & & $\mathrm{M}$ & * animale & 245 & $\mathrm{~K}$ & & & & * indécente \\
\hline $192 \mathrm{~K}$ & $\mathrm{~K}$ & & & * anticipée & 246 & & $\mathrm{C}$ & & $\mathrm{M}$ & * indescriptible \\
\hline 193 & $\mathrm{C}$ & & & * ardente & 247 & $\mathrm{~K}$ & $\mathrm{C}$ & $\mathrm{T}$ & & * indicible \\
\hline 194 & $\mathrm{C}$ & & & * austère & 248 & $\mathrm{~K}$ & $\mathrm{C}$ & $\mathrm{T}$ & & * ineffable \\
\hline 195 & & & M & * brusque & 249 & & $\mathrm{C}$ & & $\mathrm{M}$ & * inépuisable \\
\hline $196 \mathrm{~K}$ & $\mathrm{C}$ & $\mathrm{T}$ & M & * bruyante & 250 & & C & $T$ & & * inexprimable \\
\hline $197 \mathrm{~K}$ & & & & * cachée & 251 & & & $T$ & & * infernale \\
\hline 198 & $\mathrm{C}$ & & M & * calme & 252 & & $\mathrm{C}$ & $\mathrm{T}$ & $\mathbf{M}$ & * infinie \\
\hline 199 & & $\mathrm{~T}$ & M & * céleste (iitt.) & 253 & & $\mathrm{C}$ & & & * insigne \\
\hline 200 & & & $\mathbf{M}$ & * collective & 254 & $\mathrm{~K}$ & $\mathrm{C}$ & & & * insolente \\
\hline 201 & $\mathrm{C}$ & & & * communicative & 255 & & $\mathrm{C}$ & & & * intarissables \\
\hline $202 \mathrm{~K}$ & $\mathrm{C}$ & & & * complète & 256 & $\mathrm{~K}$ & $\mathrm{C}$ & & $\mathrm{M}$ & * intense \\
\hline 203 & & & $\mathbf{M}$ & * constante & 257 & & $\mathrm{C}$ & & $\mathrm{M}$ & * intéricure \\
\hline 204 & $\mathrm{C}$ & & M & $*$ courte & 258 & & $\mathrm{C}$ & $\mathbf{T}$ & & * intime \\
\hline 205 & & $T$ & M & * cruelie & 259 & $\mathrm{~K}$ & C & & & * maligne \\
\hline 206 & & & $\mathbf{M}$ & * débordante & 260 & & & $\mathrm{~T}$ & & * malsaine \\
\hline 207 & & $\mathrm{~T}$ & & * délicieuse & 261 & $\mathrm{~K}$ & & $\mathrm{~T}$ & $\mathbf{M}$ & * mauvaise \\
\hline $208 \mathrm{~K}$ & $\mathrm{C}$ & $\mathrm{T}$ & M & * délirante & 262 & & & & $\mathrm{M}$ & * méchante \\
\hline 209 & & $\mathrm{~T}$ & & * démoniaque & 263 & & & & $\mathrm{M}$ & * mêlée de larmes \\
\hline 210 & & $\mathrm{~T}$ & & * du diable & 264 & & $\mathrm{C}$ & & & menues * \\
\hline 211 & & $\mathrm{~T}$ & & * divine & 265 & $\mathrm{~K}$ & $\mathrm{C}$ & & M & * muette \\
\hline 212 & & & $\mathbf{M}$ & $*$ douce & 266 & & & & $\mathrm{M}$ & * paradisiaque (litt) \\
\hline 213 & & & M & $*$ durable & 267 & & $\mathrm{C}$ & $\mathrm{T}$ & & * parfaite \\
\hline 214 & & & M & * dure & 268 & & $\mathrm{C}$ & & $\mathrm{M}$ & * passagère \\
\hline 215 & & & $\mathrm{M}$ & * éclatante & 269 & & & & $\mathrm{M}$ & * perverse \\
\hline $216 \mathrm{~K}$ & $x$ & & & * effrénée & 270 & & $\mathrm{C}$ & $\mathrm{T}$ & & petite(s)* \\
\hline 217 & & & M & * énivrante & 271 & & & $\mathrm{~T}$ & & * populaire \\
\hline 218 & & & M & * enthousiaste & 272 & $\mathrm{~K}$ & $\mathrm{C}$ & & M & * profonde \\
\hline 219 & $\mathrm{C}$ & & & * ephémère & 273 & & $\mathrm{C}$ & & & * puérile \\
\hline 220 & & $\mathrm{~T}$ & & * esthétique & 274 & & C & $\mathrm{T}$ & & * pure \\
\hline 221 & & $\mathrm{~T}$ & & * eternelle & 275 & & C & $\mathrm{T}$ & & * radieuse \\
\hline $222 \mathrm{~K}$ & $\mathrm{C}$ & & & * étudiée & 276 & & $\mathrm{C}$ & & & * rayonnante \\
\hline 223 & & & $M$ & * exaltante & 277 & & & & M & * sadique \\
\hline $224 \quad \mathrm{~K}$ & $\mathrm{C}$ & & & * excessive & 278 & & & $\mathrm{~T}$ & & * sans bornes \\
\hline 225 & $\mathrm{C}$ & & & * extatique & 279 & & & $\mathrm{~T}$ & & * sans limite \\
\hline 226 & $\mathrm{C}$ & & & * extraordinaire & 280 & & $\mathrm{C}$ & $\mathrm{T}$ & $\mathrm{M}$ & * sans mélange \\
\hline 227 & $\mathrm{C}$ & $\mathrm{T}$ & & * extrềme & 281 & $\mathrm{~K}$ & $\mathrm{C}$ & & & * satanique \\
\hline $228 \mathrm{~K}$ & $\mathrm{C} C$ & & M & * exubérante & 282 & $\mathrm{~K}$ & & $\mathrm{~T}$ & M & * secrète \\
\hline $229 \mathrm{~K}$ & $\mathrm{C}$ & & & fausse * & 283 & & & $\mathrm{~T}$ & & * sensuelle \\
\hline 230 & $\mathrm{C}$ & & & $*$ feinte & 284 & $\mathrm{~K}$ & $\mathrm{C}$ & & $\mathbf{M}$ & * sereine \\
\hline 231 & $\mathrm{C}$ & $\mathrm{T}$ & M & * féroce & 285 & & & $\mathrm{~T}$ & & * sexuelle \\
\hline $232 \mathrm{~K}$ & $\mathrm{C}$ & $\mathrm{T}$ & & $*$ folle & 286 & & & $\mathrm{~T}$ & & * simple \\
\hline 233 & & & M & * furtive & 287 & & & $\mathrm{~T}$ & & * sombre \\
\hline 234 & $\mathrm{C}$ & & & * générale & 288 & & & & $\mathbf{M}$ & * subite \\
\hline 235 & $\mathrm{C}$ & $\mathrm{T}$ & $\mathbf{M}$ & grande * & 289 & & $\mathrm{C}$ & & & * tendre \\
\hline 236 & & $T$ & & grosse * & 290 & & & $\mathrm{~T}$ & & $*$ terrible \\
\hline 237 & & $\mathrm{~T}$ & & * horrible & 291 & & & $\mathrm{~T}$ & & * tonitruante \\
\hline $238 \mathrm{~K}$ & & $\mathrm{~T}$ & M & * immense & 292 & & & $\mathrm{~T}$ & & $*$ totale \\
\hline 239 & $\mathrm{C}$ & & & * immodérée & 293 & & & & M & * tranquille \\
\hline 240 & & & $\mathbf{M}$ & * imprévue & 294 & & & $\mathrm{~T}$ & & * triviale \\
\hline 241 & & $\mathrm{~T}$ & $\mathrm{M}$ & * inaltérable & 295 & & & & $M$ & $*$ trouble \\
\hline 242 & & & M & * inattendue & 296 & & & & M & * tumultueuse \\
\hline 243 & $\mathrm{C}$ & & & * incomparable & 297 & & $\mathrm{C}$ & & M & * turbulente \\
\hline
\end{tabular}


298

299

C) Collocations de type $N+$ de joie :

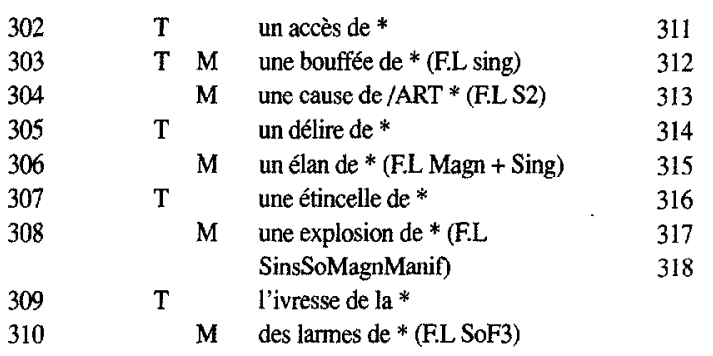

$$
T^{M} \quad \begin{aligned}
& * \text { vive } \\
& \text { vrai } *
\end{aligned}
$$

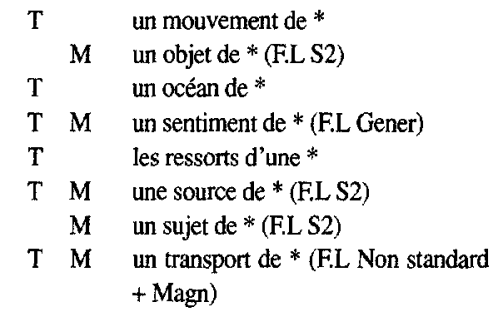

\subsection{Le tournoi et ses règles}

Tournoi pour l'accommodement des dictionnaires de collocations : le titre est provocateur. Il suggère que ces répertoires ne seraient aujourd'hui point commodes en suffisance. Et, de fait, des quatre dictionnaires pris en référence et que toujours nous consultons avec amour, nous avons fait - qui aime bien châtie bien - nos souffre-douleur: celui-ci $(T L F)$ ne fait pas partie de la famille...; celui-là $(K F D)$ alphabétise, mais lasserait l'œil en cas de kyrielles populeuses...; cet autre $(D C F C$ ) fonctionne en cycles, mais y admet par force maints disparates; cet autre enfin $(D E C)$ attache sa charrue aux étoiles, mais on peine à le sillonner tout en craignant que les astres jamais n'y foisonnent assez...

Enfin quoi, clameront à bon droit les auteurs, on nous prend pour des têtes de Turc? Nous espérons qu'ils voudront bien excuser nos chicaneries. Après tout, nulle autre ferveur ne nous mène que la leur même: un principe de progrès, auquel beaucoup encore sont attachés. Plus précisément: nous postulons qu'il est possible de mieux dire les collocations de JOIE qu'il a été fait jusqu'ici. D'où l'idée d'ouvrir en la matière un concours en vue de choisir... le «mieux-disant lexicographique». Voici donc les règles du tournoi annoncé:

\section{Article 1:}

L'objectif est pratique. Il s'agit d'organiser les collocations françaises de JOIE de façon que le consultant atteigne vite et sans peine le peloton des plus propres d'entre elles à satisfaire ses besoins rédactionnels du moment.

Article 2:

Par convention, le consultant sera considéré comme un étranger connaissant passablement le français, et en train d'exécuter une tâche de traduction vers cette langue ou de rédiger directement dans cette langue. On peut aussi imaginer ce consultant comme de langue maternelle française mais peinant à remobiliser, lors d'une rédaction dans sa langue, la ou les collocations voulues.

\section{Article 3:}

Par convention, et quoi qu'ils en aient, les concurrents voudront bien s'astreindre à tenir comme collocations françaises de JOIE toutes les combinaisons numérotées supra au $\$ 3.1$., et rien qu'elles. De toutes, et d'elles seulement, ils rendront compte en leur épreuve. Il entre de l'arbitraire dans cette convention: on s'y soumettra néanmoins, pour simplifier la tâche du jury chargé d'évaluer les résultats.

Article 4:

Les concurrents voudront bien remettre au jury, par écrit, obligatoirement une épreuve, facultativement une glose. 
Article 5:

L'épreuve, obligatoire, vise à réorganiser les données du $§ 3.1$. Celles-ci se présentent actuellement sous forme de trois listes contrastant - sur critère de partie-du-discours collocations dites verbales, collocations dites adjectivales, et collocations de type $N+d e$ joie, chaque liste étant alphabétisée sur le mot lourd de la collocation. Il va s'agir de redistribuer ces données de la manière suivante:

- Le premier critère de classement sera d'ordre sémantique. À cette fin, les concurrents s'inspireront autant, et aussi avant qu'il est possible, du modèle à archilexèmes majusculisés suggéré par F. J. Hausmann dans Encycl. Int. de Lexicogr. (op. cit.; v. supra, § 1. [5]). Les concurrents peuvent s'inspirer aussi des catégories sémantiques (notions, actants sémantiques) sous-jacentes à la notation des fonctions lexicales du DEC: en ce cas, il leur est conseillé d'en simplifier l'économie et d'en imaginer une reformulation qui, tout en demeurant concise, soit conviviale avant tout, c'est-à-dire accessible au consultant sans un apprentissage exorbitant. Bref, il s'agit de distribuer les collocations par petits groupes et d'étiqueter ces groupes de manière assez précise pour guider l'esprit et assez discrète pour ne pas l'empêtrer: un moyen terme en somme - pour ce qui est des notations - entre l'aridité du $D C F C$ et le pullulement du $D E C$.

- Au plan macrosémantique, les concurrents sont libres de tenir que le vocable JOIE n'a qu'un seul sens, qu'il en a deux (comme dans le $D C F C$ ), ou qu'il en a trois (comme dans le $D E C$ ). Dans les deux dernières hypothèses, ils distribueront en conséquence les collocations sous deux ou trois lexèmes distincts en première hiérarchie du classement: JOIE 1 , JOIE 2 , voire JOIE 3 .

- Des critères syntaxiques pourront, bien entendu avoir un tôle dans le classement proposé.

- Le critère de partie-du-discours (collocatif verbal $\neq$ collocatif adjectif $\neq$ collocatif $N+$ de) pourra être considéré comme principal ou comme accessoire. Dans le second cas, on ne conservera donc pas la tripartition initiale des données.

- Le critère alphabétique, enfin, ne devra plus jouer, lui aussi, qu'un rôle mineur, par exemple, lorsqu'on voudra localement ordonner des sous-groupes de collocations.

- Chaque collocation sera reproduite précédée du numéro qui lui est céans affecté au $\$ 3.1$., ce à des fins de repérage au moment de l'évaluation des épreuves.

Article 6:

La glose, facultative, permettra aux concurrents de livrer tout document complémentaire qu'ils souhaiteraient : commentaire discutant la présence de telle collocation parmi les données imposées; proposition pour l'inclusion d'autres collocations n'y figurant point encore (ex : la joie demeure; se donner la joie (de)); discussion des catégories structurales avancées par F.-J. HAUSMANN (v. supra, \$1., [2] ; justification des traitcments proposés; reprise du travail exposé dans la partie épreuve, avec adjonction d'exemples, voire de traductions à divers niveaux (traduction du vocable, des collocations, des exemples, des archilexèmes qui balisent les distributions et qui en manifestent les critères sémantiques) dans le cadre d'un projet dictionnairique bilingue; traitement d'autres vocables; projet organisationnel sous forme de base de données, etc.

Article 7:

Le concours est ouvert à tout lecteur du présent numéro de Meta - traducteurs, rédacteurs, maîtres, étudiants (en guise de T.P.), amateurs ou professionnels de la chose langagière, agissant isolément ou en équipe. En cas de publication, l'anonymat des concurrents sera respecté s'ils le souhaitent. Qu'ils veuillent bien, en cette perspective, adopter un pseudonyme (personnel ou collectif) permettant référence.

Article 8:

Un jury de rédacteurs-traducteurs sera constitué à la diligence du Comité de rédaction de la revue Meta, et sera chargé de l'évaluation des travaux. Des prix seront attribués aux lauréats $\mathrm{du}$ tournoi, sous forme de publication en cette revue, d'abonnements ou d'exemplaires offerts en hommage - Meta's kingdom for cute lists ! 
Article 9:

Le concours sera clos le 30 novembre 1995. À cette date, épreuves et gloses devront avoir été postées, le tampon de la poste faisant foi, à: Professeur André Clas, revue Meta, Université de Montréal, Département de linguistique et de traduction, C.P. 6128, succ. Centreville, Montréal, H3C 3J7, Canada.

\section{Notes}

1. Données et résultats en ont été distribués sur plusieurs numéros: Buzon, C. et al., «Un exercice dictionnairique: (I) les données du problèmes», C.L. $\mathrm{n}^{\circ} 36,1980-1: 110-118$; Buzon, C. et al., «Un exercice dictionnairique : (II) solutions et discussions», C.L. $\mathrm{n}^{\circ}$ 38, 1981-1: 60-99; Buzon, C. et al., «Un exercice dictionnairique (Complement)», $\mathrm{n}^{\circ} 40,1982-1: 113-115$; Elnitsky L. «Une description du verbe flamber : exercice dictionnairique», C.L. $\mathrm{n}^{\circ} 40,1982-1: 95-111$.

2. Encyclopédie internationale de lexicographie, Hausmann, F.-J. et al. Eds, W. de Gruyter, Berlin-New York, vol. $1,1989$.

3. Hausmann, F.-J. (1989), «Le dictionnaire de collocations», Encycl. internat. de lexicogr., W. de Gruyter, Berlin, vol 1: 1010-1019.

4. V. dans le même esprit : Bardosi, V., «La rédaction d'un dictionnaire onomasiologique de locutions : esquisse d'une problématique», Annales universitatis Budapestinensis, Sectio Philologica Moderna, $\mathrm{n}^{\circ} 13 ; 1982$ : 21-34 ; et Bardosi, V., Csink L., «Le traitement des locutions idiomatiques par micro-ordinateur», Acta Universitatis Szegedinensis, Studia Lexicographica neolatina, 1986: 10-51.

5. Trésor de la langue française du 19 e et du 20e, Centre Nat. de la Rech. Scient., Gallimard, Paris, [16 vols], vol. 10, 1983.

6. Kontextwörterbuch französisch-deutsch [dictionnaire de collocations français-allemand; auteur principal: Ilgenfritz, P.], Langenscheidt, 1989.

7. Dictionnaire de collocations français-chinois [auteur principal: Liang, S. Q., Univ. Zhongshan, GuangZhou], s. - presse.

8. Dictionnaire explicatif et combinatoire du français contemporain, recherches lexico-sémantiques [auteur principal: Mel'čuk, I. A.], Les Presses de l'Université de Montréal, [3 vols publ.], vol. 1, 1984: 172 p.; vol. $2,1988: 332$ p.; vol. $3,1991: 332$ p.

9. Liang, S. Q. (1991), «À propos du Dictionnaire français-chinois des collocations françaises», Cahiers de Lexicologie, 1991-2, $\mathrm{n}^{\circ} 59: 151-167$.

10. À titre de comparaison, précisons que, sous le vocable JOIE, le $D C F C$ distingue deux lexèmes seulement, et le $K F D$ n'en distingue pas. Distinguer ou non plusieurs lexèmes sous un vocable est un problème délicat, en lexicographie collocationnelle comme en lexicographie générale.

11. V. aussi, dans la mouvance du $D E C$, le stimulant programme lexicographique français-espagnol / espagnolfrançais, proposé par B. Lépinette sous le titre : "Vers un dictionnaire explicatif et combinatoire bilingue», Cah. de lex. ${ }^{\circ} 54,1989-1: 105-162$.

12. Proust, M. (1922), La Prisonnière, ALRDTP, Gallimard, 1954, t. III, p. 265 (note).

13. Extrait du $D E C$ : JOIE 1 [Liste des fonctions lexicales (fragment)]

\begin{tabular}{|c|c|}
\hline $\begin{array}{l}\text { IncepPredMinus } \\
\text { CausPredMinus }\end{array}$ & $\begin{array}{l}\text { : diminuer, faiblif } \\
\text { : diminuer [ÁRT } ~-\text { ] }\end{array}$ \\
\hline Bon & : suns malange \\
\hline Magn + Bon & $\begin{array}{l}\text { : Ifit cetteste, litt paradisiaque; litt du } \\
\text { ccour, litt de l'âme }\end{array}$ \\
\hline $\begin{array}{l}\text { Magntemp + Bon } \\
X \text { n'est pas excité }+\end{array}$ & : inaltérable \\
\hline Bon & : douce, calme, tranquilte, sereine \\
\hline AntiBon & : melke de larmes; trouble, amère \\
\hline CausPredPejor & : alterer, gicher [ART - ] \\
\hline$A d v_{1}$ & $\begin{array}{l}\text { dans un moment [de - ] [Dans un } \\
\text { moment de grande joie (de joie indi- } \\
\text { cible) elle se décida de tout lui } \\
\text { owouer]; dans [ART - ] [Dans so } \\
\text { joie Andre a oublie de..]: avec } \\
\text { [Ø/ART ] }\end{array}$ \\
\hline nonAdv $v_{1}$ & $:$ sans [D/ART -1 \\
\hline$A_{d v}$ & $\begin{array}{l}\text { : a ha } \sim \| C_{1} \neq A[\dot{A} \text { la grande jaie } \\
\text { de Marie. son fils a néresi lexamen] }\end{array}$ \\
\hline Propt & 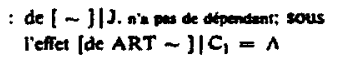 \\
\hline
\end{tabular}

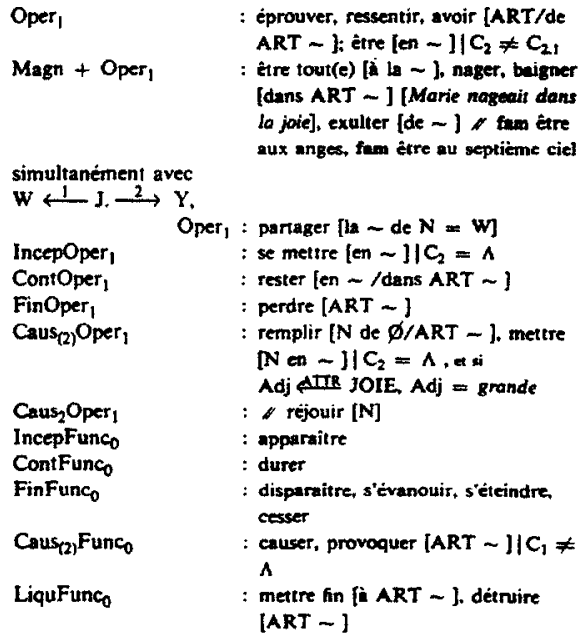

